\title{
Fuzzy Time Series Forecasting Model Based on Intuitionistic Fuzzy Sets via Delegation of Hesitancy Degree to the Major Grade De-i-fuzzification Method
}

\author{
Nik Muhammad Farhan Hakim Nik Badrul Alam, Nazirah Ramli", Norhuda Mohammed \\ Faculty of Computer and Mathematical Sciences, Universiti Teknologi MARA Pahang, 26400 Bandar Jengka, Pahang, Malaysia
}

Received November 24, 2020; Revised January 23, 2021; Accepted February 17, 2021

\section{Cite This Paper in the following Citation Styles}

(a): [1] Nik Muhammad Farhan Hakim Nik Badrul Alam, Nazirah Ramli, Norhuda Mohammed, "Fuzzy Time Series Forecasting Model Based on Intuitionistic Fuzzy Sets via Delegation of Hesitancy Degree to the Major Grade De-i-fuzzification Method," Mathematics and Statistics, Vol. 9, No. 1, pp. 46 - 53, 2021. DOI: 10.13189/ms.2021.090108.

(b): Nik Muhammad Farhan Hakim Nik Badrul Alam, Nazirah Ramli, Norhuda Mohammed (2021). Fuzzy Time Series Forecasting Model Based on Intuitionistic Fuzzy Sets via Delegation of Hesitancy Degree to the Major Grade De-i-fuzzification Method. Mathematics and Statistics, 9(1), 46 - 53. DOI: 10.13189/ms.2021.090108.

Copyright $\bigcirc 2021$ by authors, all rights reserved. Authors agree that this article remains permanently open access under the terms of the Creative Commons Attribution License 4.0 International License

\begin{abstract}
Fuzzy time series is a powerful tool to forecast the time series data under uncertainty. Fuzzy time series was first initiated with fuzzy sets and then generalized by intuitionistic fuzzy sets. The intuitionistic fuzzy sets consider the degree of hesitation in which the degree of non-membership is incorporated. In this paper, a fuzzy set time series forecasting model based on intuitionistic fuzzy sets via delegation of hesitancy degree to the major grade de-i-fuzzification approach was developed. The proposed model was implemented on the data of student enrollments at the University of Alabama. The forecasted output was obtained using the fuzzy logical relationships of the output, and the performance of the forecasted output was compared with the fuzzy time series forecasting model based on fuzzy sets using the mean square error, root mean square error, mean absolute error, and mean absolute percentage error. The results showed that the forecasting model based on induced fuzzy sets from intuitionistic fuzzy sets performs better compared to the fuzzy time series forecasting model based on fuzzy sets.
\end{abstract}

Keywords De-i-fuzzification, Forecasting Enrollment, Fuzzy Time Series, Hesitancy Degree, Induced Fuzzy Set, Intuitionistic Fuzzy Set

\section{Introduction}

Fuzzy time series was first introduced by Song and
Chissom [1] and it was applied to the data of student enrollments at the University of Alabama [2]. After that, many researchers have developed and improved the fuzzy time series forecasting model by modifying the interval lengths, the order of the fuzzy logical relationships, and the rules for calculating the forecasted output [3-10]. Fuzzy forecasting is better than other forecasting methods since it can handle unclear and uncertain data [11].

However, the aforementioned models did not consider the degree of hesitation in their forecasting methods. In 1986, Atanassov [12] first introduced the concept of intuitionistic fuzzy sets, which incorporates the degree of membership and non-membership of the fuzzy sets. Castillo et al. [13] applied the intuitionistic fuzzy sets in the fuzzy time series by applying them to plant monitoring and diagnosis. Since then, many researchers have developed the fuzzy time series forecasting model based on intuitionistic fuzzy sets such as Joshi and Kumar [14], Gangwar and Kumar [15], Kumar and Gangwar [16], Fan et al. [17], Bisht et al. [18] and, Gupta and Kumar [19]. In a study by Joshi and Kumar [14], an algorithm for fuzzy time series forecasting, which includes the hesitation index in establishing a fuzzy logical relationship was introduced. Meanwhile, Kumar and Gangwar [16] used the fuzzy set induced by the intuitionistic fuzzy set to establish the fuzzy time series forecasting model. Bisht et al. [18] then introduced the application of dual hesitant fuzzy sets in intuitionistic fuzzy set forecasting to handle non-determinism that occurs due to multiple valid 
fuzzification methods for time series data. While Abhishekh et al. [20] proposed the score function for intuitionistic fuzzifying of the historical time series data, Bisht and Kumar [21], recently, have also proposed an intuitionistic fuzzy set with a simplified algorithm in financial prediction.

A process of converting an intuitionistic fuzzy set into a fuzzy set is one of the core procedures in the fuzzy time series forecasting model based on the intuitionistic fuzzy set, which was termed as the de-i-fuzzification process by Attanassova [22] and Ban et al. [23]. During this process, the hesitancy degree is reduced to zero, and the summation of membership and non-membership degree becomes one. Ansari et al. [24] also introduced three different methods of de-i-fuzzification, which are assigning hesitancy to the major grade, equal distribution of hesitancy, and proportionate allocation.

In this paper, we propose the fuzzy time series forecasting model based on fuzzy sets induced from intuitionistic fuzzy sets using the de-i-fuzzification approach by assigning the degree of hesitancy to the major grade. The performance of the proposed method was compared with the fuzzy time series forecasting based on fuzzy sets.

This paper is organized as follows: Section 2 reviews the fuzzy time series and intuitionistic fuzzy sets; Section 3 presents the proposed fuzzy time series model based on induced fuzzy sets; Section 4 implements the proposed model on student enrollments at the University of Alabama; Section 5 presents and discusses the forecasted output; and Section 6 concludes the paper.

\section{Review of Fuzzy Time Series and Intuitionistic Fuzzy Sets}

In this section, some concepts of fuzzy sets, fuzzy time series, and intuitionistic fuzzy sets are reviewed.

Definition 2.1: If $S$ is a fuzzy set on the universe of discourse, $D=\left\{d_{1}, d_{2}, d_{3}, \ldots, d_{n}\right\}$, then $\mathrm{D}$ can be written as

$$
D=\mu_{S}\left(d_{1}\right) / d_{1}+\mu_{S}\left(d_{2}\right) / d_{2}+\mu_{S}\left(d_{3}\right) / d_{3}+\ldots+\mu_{S}\left(d_{n}\right) / d_{n}
$$

where $\mu_{S}\left(d_{i}\right)$ is the membership grade of the elements $d_{i} \in D$ for $i=1,2,3, \ldots, n$.

Definition 2.2: If $Y(t),(t=0,1,2, \ldots)$ is the universe of discourse and fuzzy sets $f_{i}(t),(i=0,1,2, \ldots)$ are defined on $Y(t)$, then the fuzzy time series of $Y(t)$ is a collection of fuzzy sets $f_{i}(t)$.

Definition 2.3: If only $Y(t)$ affects $F(t)$, then $F(t)=F(t-1) \circ R(t, t-1)$ represents $F(t-1) \rightarrow F(t)$, where $R(t, t-1)$ is a fuzzy relation between $F(t)$ and
$F(t-1)$.

Definition 2.4: An intuitionistic fuzzy set $I$ in $D$ can be written in the form

$$
I=\left\{\left\langle x, \mu_{I}(x), v_{I}(x)\right\rangle: x \in D\right\}
$$

where the functions $\mu_{I}(x): D \rightarrow[0,1]$ and $v_{I}(x): D \rightarrow[0,1]$ are the degree of membership and non-membership of $x$ in $D$, respectively. For every $x$ in $D$,

$$
0 \leq \mu_{I}(x)+v_{I}(x) \leq 1 .
$$

The degree of indeterminacy is defined by $\pi_{I}(x)=1-\mu_{I}(x)-v_{I}(x) . \quad \pi_{I}$ is also known as the degree of hesitancy of $x$ in $D$.

Definition 2.5: Let $\beta \in \kappa$ where $\kappa$ is the collection of all fuzzy sets in $D$. Let $\alpha: D \rightarrow[0,1]$ and $\beta: D \rightarrow[0,1]$. $f:[0,1]^{2} \times[0,1] \rightarrow L^{*}$, where $f(x, \alpha, \beta)=\left(f_{\mu}(x, \alpha, \beta)\right.$, $f_{v}(x, \alpha, \beta)$ and

$$
\begin{gathered}
f_{\mu}(x, \alpha, \beta)=x(1-\alpha \beta), \\
f_{v}(x, \alpha, \beta)=1-x(1-\alpha \beta)-\alpha \beta .
\end{gathered}
$$

\subsection{De-i-fuzzification Process by Assigning Hesitancy to Major Grade [24]}

Ansari et al. [24] proposed a de-i-fuzzification process by assigning the degree of hesitancy to the major grade of membership or non-membership. The proposed de-i-fuzzification process is given as follows:

Let $\pi$ be the degree of hesitancy and the intuitionistic fuzzy set is given as $I=\langle x, \mu(x), v(x)\rangle$. If the degree of membership $\mu$ is greater than the degree of non-membership $v$, then the degree of membership of the fuzzy set is $\mu+\pi$. However, if the degree of membership $\mu$ is less than the degree of non-membership $v$, then the degree of membership of fuzzy set remains as $\mu$.

\section{Proposed Fuzzy Time Series Forecasting Model Based on Fuzzy Sets and IFSs}

In this section, we will explain the following ten steps of the proposed fuzzy time series forecasting model in detail:

Step 1: Define the universe of discourse, $D=\left[D_{\min }-D_{a}, D_{\max }+D_{b}\right]$ where $D_{\min }$ and $D_{\max }$ are the minimum and maximum values of the historical data, while $D_{a}$ and $D_{b}$ are two suitable positive integers.

Step 2: Partition $D$ into a certain number of intervals using the frequency density-based method [25].

Step 3: Fuzzify the historical data using triangular fuzzy numbers. 
Step 4: Convert the fuzzy sets into intuitionistic fuzzy sets using Definition 2.5.

Step 5: De-i-fuzzify the intuitionistic fuzzy sets into fuzzy sets by assigning the degree of hesitancy to major grade [24].

Step 6: Obtain the induced fuzzy sets by considering the newly obtained membership values only.

Step 7: Establish the fuzzy logical relationships from the fuzzy sets obtained in Step 3 and induced fuzzy sets obtained in Step 6. Suppose the fuzzy logical relationships obtained are $\tilde{F}_{a} \rightarrow \tilde{F}_{b}, \tilde{F}_{c} \rightarrow \tilde{F}_{d}, \ldots, \tilde{F}_{y} \rightarrow \tilde{F}_{z}$, then a matrix $R$ can be obtained by the relation $\tilde{F}_{a}^{T} \times \tilde{F}_{b} \cup \tilde{F}_{c}^{T} \times \tilde{F}_{d} \cup \ldots \cup \tilde{F}_{y}^{T} \times \tilde{F}_{z}$.

Step 8: Perform the max-min composition operation on the matrix obtained in Step 7 to obtain the fuzzified forecasted data as $A_{i}=A_{i-1} \circ R$, whereby $A_{i}$ and $A_{i-1}$ are the forecasted data at year $i$ and $i-1$, respectively, and $\circ$ is the max-min composition operator.

Step 9: From the fuzzified forecasted data, obtain the fuzzy logical relationships.

Step 10: Calculate the forecasted output using the fuzzy logical relationships obtained in Step 9.

The arithmetic rules for calculating the forecasted output are as follows:

1. If the fuzzified data of year $n$ is $\tilde{F}_{a}$ and there is a unique fuzzy logical relationship, for instance $\tilde{F}_{a} \rightarrow \tilde{F}_{b}$, where the membership value of $\tilde{F}_{b}$ occurs in $D_{b}=\left[T_{b 1}, T_{b 2}\right]$, then the forecasted enrolment of year $n+1$ is given by

$$
\frac{T_{b 1}+T_{b 2}}{2} \text {. }
$$

2. If the fuzzified data of year $n$ is $\tilde{F}_{a}$ and there are $p$ fuzzy logical relationships, say $\tilde{F}_{a} \rightarrow \tilde{F}_{b}$, $\tilde{F}_{a} \rightarrow \tilde{F}_{c}, \ldots, \tilde{F}_{a} \rightarrow \tilde{F}_{k}$, where the membership values of $\tilde{F}_{b}, \tilde{F}_{c}, \ldots, \tilde{F}_{k}$ occur in $D_{b}=\left[T_{b 1}, T_{b 2}\right]$ $D_{c}=\left[T_{c 1}, T_{c 2}\right] \ldots, D_{k}=\left[T_{k 1}, T_{k 2}\right]$ respectively, then the forecasted output of year $n+1$ is given by

$$
\frac{T_{b 1}+T_{b 2}+T_{c 1}+T_{c 2}+\ldots+T_{k 1}+T_{k 2}}{2 p} .
$$

3. If the fuzzified data of year $n$ is $\tilde{F}_{a}$ whose membership value occurs in $D_{a}=\left[T_{a 1}, T_{a 2}\right]$ and there are no fuzzy logical relationships, then the forecasted data of year $n+1$ is given by

$$
\frac{T_{a 1}+T_{a 2}}{2} \text {. }
$$

The above steps are illustrated in the following flowchart:

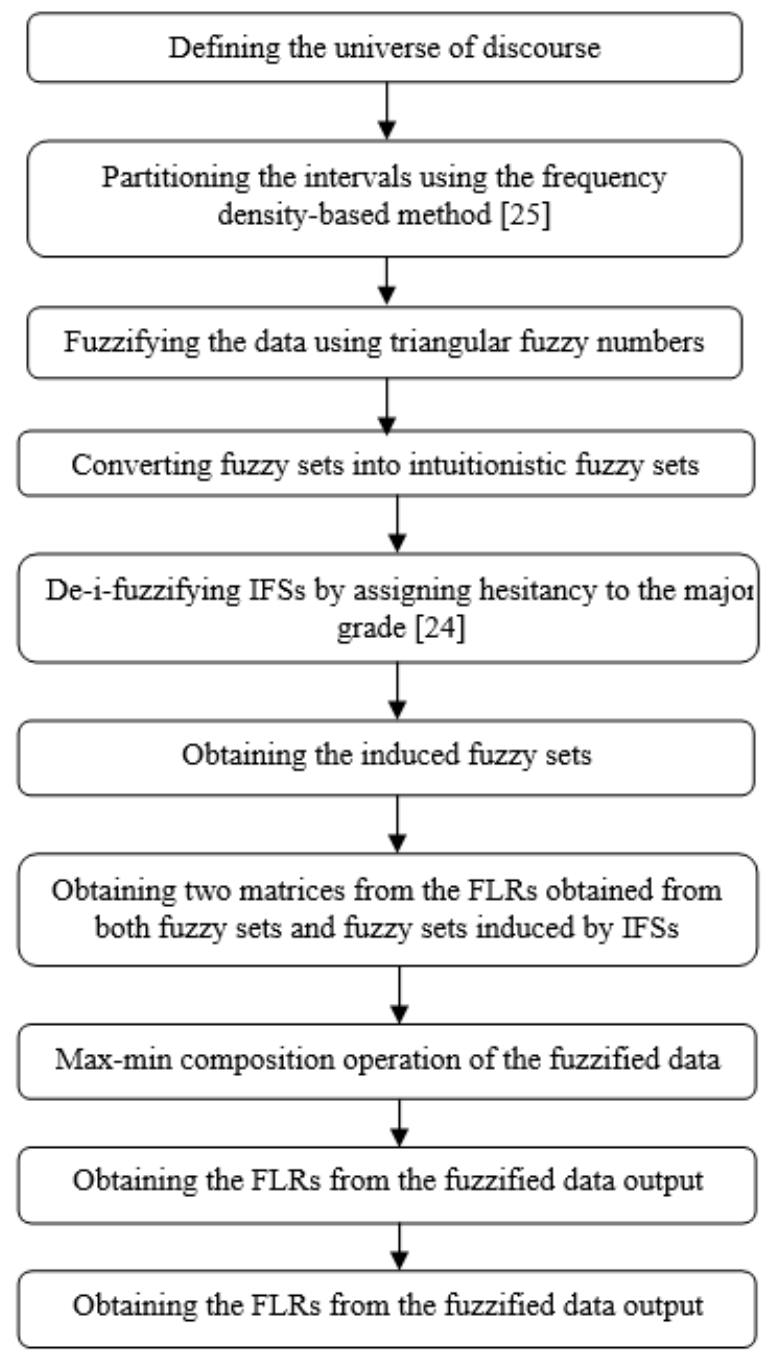

Figure 1. Steps of the proposed model

\section{Forecasting Student Enrollments at the University of Alabama}

Figure 2 presents the historical data of student enrollments at the University of Alabama from 1971 until 1992 as adopted from Song and Chissom [2-3]. 


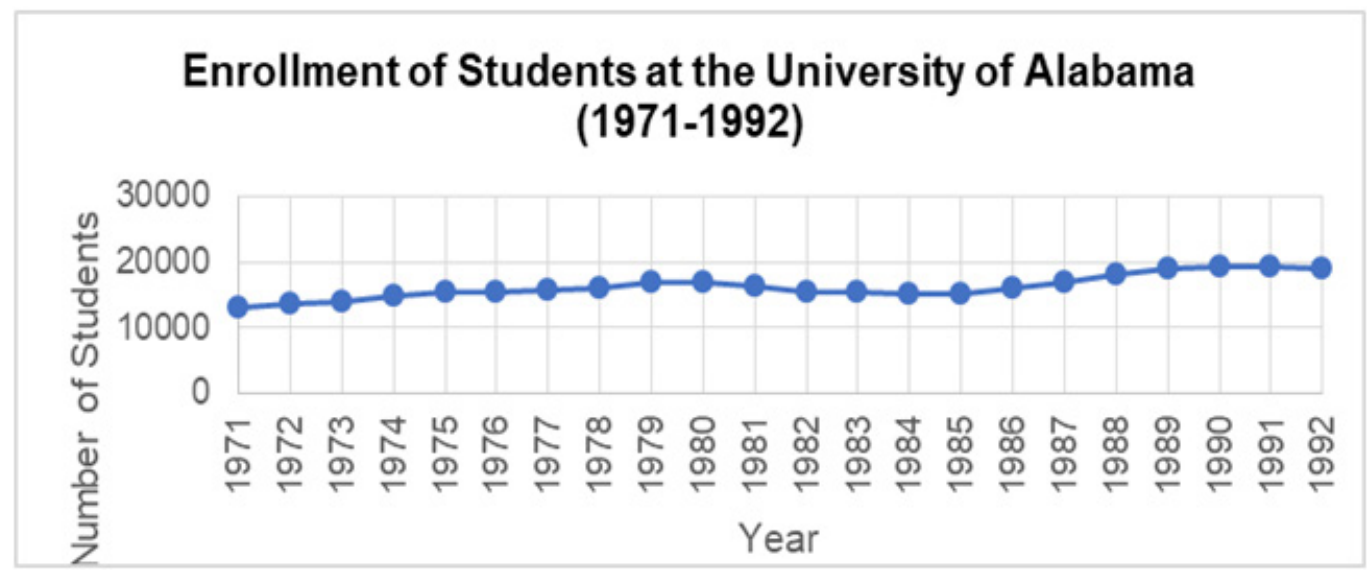

Figure 2. Student Enrollments at the University of Alabama (1971-1992)

The minimum and maximum data extracted based on Figure 2 are 13055 and 19337, respectively. The universe of discourse was defined by $D=[13055-55,19337+663]=$ $[13000,20000]$. Then, $D$ was divided using the frequency density-based method [25] and 14 intervals were obtained. The triangular fuzzy sets corresponding to these intervals are shown in Table 1.

Table 1. Triangular fuzzy sets corresponding to the intervals.

\begin{tabular}{|c|c|}
\hline Intervals & Triangular Fuzzy Sets \\
\hline $\mathrm{D}_{1}=[13000,13500]$ & $\mathrm{E}_{1}=(13000,13500,14000)$ \\
\hline $\mathrm{D}_{2}=[13500,14000]$ & $\mathrm{E}_{2}=(13500,14000,15000)$ \\
\hline $\mathrm{D}_{3}=[14000,15000]$ & $\mathrm{E}_{3}=(14000,15000,15250)$ \\
\hline $\mathrm{D}_{4}=[15000,15250]$ & $\mathrm{E}_{4}=(15000,15250,15500)$ \\
\hline $\mathrm{D}_{5}=[15250,15500]$ & $\mathrm{E}_{5}=(15250,15500,15750)$ \\
\hline $\mathrm{D}_{6}=[15500,15750]$ & $\mathrm{E}_{6}=(15500,15750,16000)$ \\
\hline $\mathrm{D}_{7}=[15750,16000]$ & $\mathrm{E}_{7}=(15750,16000,16333)$ \\
\hline $\mathrm{D}_{8}=[16000,16333]$ & $\mathrm{E}_{8}=(16000,16333,16667)$ \\
\hline $\mathrm{D}_{9}=[16333,16667]$ & $\mathrm{E}_{9}=(16333,16667,17000)$ \\
\hline $\mathrm{D}_{10}=[16667,17000]$ & $\mathrm{E}_{10}=(16667,17000,18000)$ \\
\hline $\mathrm{D}_{11}=[17000,18000]$ & $\mathrm{E}_{11}=(17000,18000,18500)$ \\
\hline $\mathrm{D}_{12}=[18000,18500]$ & $\mathrm{E}_{12}=(18000,18500,19000)$ \\
\hline $\mathrm{D}_{13}=[18500,19000]$ & $\mathrm{E}_{13}=(18500,19000,20000)$ \\
\hline $\mathrm{D}_{14}=[19000,20000]$ & $\mathrm{E}_{14}=(19000,20000,20000)$ \\
\hline
\end{tabular}

The data were then fuzzified to obtain the following fuzzy sets.

$$
\begin{aligned}
F_{1}= & 0.110 / 13055+0.874 / 13563+0.266 / 13867 \\
F_{2}= & 0.126 / 13563+0.734 / 13867+0.304 / 14696 \\
F_{3}= & 0.696 / 14696+0.420 / 15145+0.348 / 15163 \\
F_{4}= & 0.580 / 15145+0.652 / 15163+0.756 / 15311 \\
& +0.268 / 15433+0.160 / 15460+0.012 / 15497
\end{aligned}
$$

$$
\begin{gathered}
F_{5}=0.244 / 15311+0.732 / 15433+0.840 / 15460 \\
+0.988 / 15497+0.588 / 15603 \\
F_{6}=0.412 / 15603+0.556 / 15861+0.064 / 15984 \\
F_{7}=0.444 / 15861+0.936 / 15984 \\
F_{8}=0.835 / 16388 \\
F_{9}=0.165 / 16388+0.580 / 16807+0.423 / 16859 \\
+0.243 / 16919 \\
F_{10}=0.420 / 16807+0.577 / 16859+0.757 / 16919 \\
F_{11}=0.700 / 18150 \\
F_{12}=0.300 / 18150+0.248 / 18876+0.060 / 18970 \\
F_{13}=0.752 / 18876+0.940 / 18970+0.672 / 19328 \\
+0.663 / 19337 \\
\quad F_{14}=0.328 / 19328+0.337 / 19337
\end{gathered}
$$

The fuzzy sets were then converted into intuitionistic fuzzy sets using Definition 2.5. The intuitionistic fuzzy sets obtained are as follows:

$$
\begin{aligned}
I_{1}= & \{(13055,0.099,0.804),(13563,0.790,0.114), \\
& (13867,0.240,0.663)\} \\
I_{2}= & \{(13563,0.114,0.793),(13867,0.666,0.241), \\
& (14696,0.276,0.632)\} \\
I_{3}= & \{(14696,0.527,0.230),(15145,0.318,0.440), \\
& (15163,0.264,0.494)\} \\
I_{4}= & \{(15145,0.575,0.416),(15163,0.646,0.345), \\
& (15311,0.749,0.242),(15433,0.266,0.725) \\
& (15460,0.159,0.832),(15497,0.012,0.979)\} \\
I_{5}= & (15311,0.184,0.574),(15433,0.556,0.203), \\
& (15460,0.637,0.121),(15497,0.750,0.009) \\
& (15603,0.446,0.313)\}
\end{aligned}
$$




$$
\begin{aligned}
& I_{6}=\{(15603,0.397,0.567),(15861,0.536,0.428), \\
&(15984,0.062,0.903)\} \\
& I_{7}=\{(15861,0.259,0.325),(15984,0.547,0.037)\} \\
& I_{8}=\{(16388,0.253,0.050)\} \\
& I_{9}=(16388,0.149,0.755),(16807,0.524,0.380), \\
&(16859,0.383,0.522),(16919,0.220,0.685)\} \\
& I_{10}=(16807,0.286,0.396),(16859,0.394,0.289), \\
&(16919,0.516,0.166)\} \\
& I_{11}=\{(18150,0.357,0.153)\} \\
& I_{12}=(18150,0.295,0.687),(18876,0.244,0.738), \\
&(18970,0.059,0.923)\} \\
& I_{13}=((18876,0.283,0.093),(18970,0.354,0.023), \\
&(19328,0.253,0.124),(19337,0.250,0.127)\} \\
& I_{14}=\{(19328,0.292,0.598),(19337,0.300,0.590)\}
\end{aligned}
$$

The intuitionistic fuzzy sets obtained in the previous step were de-i-fuzzified by assigning the degree of hesitancy to the major grade that is $\pi_{I}(x)$, which was added to the maximum of $\mu_{I}(x)$ or $v_{I}(x)$ [24]. The induced fuzzy sets obtained are as follows:

$$
\begin{aligned}
\widetilde{F}_{1} & =0.099 / 13055+0.886 / 13563+0.240 / 13867 \\
\widetilde{F}_{2} & =0.114 / 13563+0.759 / 13867+0.276 / 14696 \\
\widetilde{F}_{3} & =0.770 / 14696+0.318 / 15145+0.264 / 15163 \\
\widetilde{F}_{4}= & 0.584 / 15145+0.655 / 15163+0.758 / 15311 \\
& +0.266 / 15433+0.159 / 15460+0.012 / 15497
\end{aligned}
$$

$$
\begin{gathered}
\widetilde{F}_{5}=0.184 / 15311+0.797 / 15433+0.879 / 15460 \\
+0.991 / 15497+0.687 / 15603 \\
\widetilde{F}_{6}=0.397 / 15603+0.572 / 15861+0.062 / 15984 \\
\widetilde{F}_{7}=0.259 / 15861+0.963 / 15984 \\
\widetilde{F}_{8}=0.950 / 16388 \\
\widetilde{F}_{9}=0.149 / 16388+0.620 / 16807+0.383 / 16859 \\
+0.220 / 16919 \\
\widetilde{F}_{10}=0.286 / 16807+0.711 / 16859+0.834 / 16919 \\
\widetilde{F}_{11}=0.847 / 18150 \\
\widetilde{F}_{12}=0.295 / 18150+0.244 / 18876+0.059 / 18970 \\
\widetilde{F}_{13}=0.907 / 18876+0.977 / 18970+0.876 / 19328 \\
+0.873 / 19337 \\
\widetilde{F}_{14}=0.292 / 19328+0.300 / 19337
\end{gathered}
$$

From the induced fuzzy set $\tilde{F}_{i}$, we listed out the fuzzy logical relationships as $\tilde{F}_{1} \rightarrow \tilde{F}_{1}, \quad \tilde{F}_{1} \rightarrow \tilde{F}_{2}, \quad \tilde{F}_{2} \rightarrow \tilde{F}_{3}$, $\tilde{F}_{3} \rightarrow \tilde{F}_{5}, \quad \tilde{F}_{5} \rightarrow \tilde{F}_{4}, \quad \tilde{F}_{4} \rightarrow \tilde{F}_{5}, \quad \tilde{F}_{5} \rightarrow \tilde{F}_{6}, \quad \tilde{F}_{6} \rightarrow \tilde{F}_{9}$, $\tilde{F}_{9} \rightarrow \tilde{F}_{10}, \tilde{F}_{10} \rightarrow \tilde{F}_{8}, \quad \tilde{F}_{8} \rightarrow \tilde{F}_{5}, \quad \tilde{F}_{5} \rightarrow \tilde{F}_{5}, \quad \tilde{F}_{4} \rightarrow \tilde{F}_{4}$, $\tilde{F}_{4} \rightarrow \tilde{F}_{7}, \quad \tilde{F}_{7} \rightarrow \tilde{F}_{10}, \quad \tilde{F}_{10} \rightarrow \tilde{F}_{11}, \quad \tilde{F}_{11} \rightarrow \tilde{F}_{13} \quad$ and $\tilde{F}_{13} \rightarrow \tilde{F}_{13}$. Then, the matrix $R=\tilde{F}_{1}^{T} \times \tilde{F}_{1} \cup \tilde{F}_{1}^{T} \times \tilde{F}_{2} \cup$ $\ldots \cup \tilde{F}_{11}^{T} \times \tilde{F}_{13} \cup \tilde{F}_{13}{ }^{T} \times \tilde{F}_{13}$ was obtained as follows:

$$
R=\left(\begin{array}{cccccccccccccc}
0.099 & 0.099 & 0.099 & 0 & 0 & 0 & 0 & 0 & 0 & 0 & 0 & 0 & 0 & 0 \\
0.099 & 0.886 & 0.759 & 0.318 & 0 & 0 & 0 & 0 & 0 & 0 & 0 & 0 & 0 & 0 \\
0 & 0 & 0.276 & 0.276 & 0.770 & 0.687 & 0 & 0 & 0 & 0 & 0 & 0 & 0 & 0 \\
0 & 0 & 0 & 0.655 & 0.655 & 0.655 & 0.655 & 0 & 0 & 0 & 0 & 0 & 0 & 0 \\
0 & 0 & 0 & 0.655 & 0.991 & 0.687 & 0.758 & 0 & 0 & 0 & 0 & 0 & 0 & 0 \\
0 & 0 & 0 & 0.655 & 0.687 & 0.687 & 0.572 & 0 & 0.149 & 0.397 & 0 & 0 & 0 & 0 \\
0 & 0 & 0 & 0 & 0 & 0 & 0 & 0 & 0.149 & 0.834 & 0 & 0 & 0 & 0 \\
0 & 0 & 0 & 0 & 0 & 0 & 0 & 0 & 0 & 0 & 0 & 0 & 0 & 0 \\
0 & 0 & 0 & 0 & 0.950 & 0.687 & 0 & 0 & 0 & 0.149 & 0 & 0 & 0 & 0 \\
0 & 0 & 0 & 0 & 0 & 0 & 0 & 0 & 0.834 & 0.620 & 0 & 0.834 & 0 & 0 \\
0 & 0 & 0 & 0 & 0 & 0 & 0 & 0 & 0 & 0 & 0 & 0 & 0 & 0 \\
0 & 0 & 0 & 0 & 0 & 0 & 0 & 0 & 0 & 0 & 0 & 0 & 0.847 & 0.847 \\
0 & 0 & 0 & 0 & 0 & 0 & 0 & 0 & 0 & 0 & 0 & 0 & 0.977 & 0.876 \\
0 & 0 & 0 & 0 & 0 & 0 & 0 & 0 & 0 & 0 & 0 & 0 & 0.876 & 0.876
\end{array}\right) .
$$

Next, we performed the max-min composition of the fuzzified enrollments for each year with $R$ to obtain the fuzzified forecasted enrollments as $A_{i}=A_{i-1} \circ R$. 
Table 2. The fuzzified forecasted enrollments after max-min composition with $R$

\begin{tabular}{|c|c|c|c|c|c|c|c|c|c|c|c|c|c|c|}
\hline Year & $D_{1}$ & $\mathbf{D}_{2}$ & $\mathbf{D}_{3}$ & $\mathbf{D}_{4}$ & $D_{5}$ & $D_{6}$ & $\mathbf{D}_{7}$ & $\mathbf{D}_{8}$ & $D_{9}$ & $D_{10}$ & $\mathbf{D}_{11}$ & $D_{12}$ & $D_{13}$ & $D_{14}$ \\
\hline 1972 & 0.099 & 0.886 & 0.759 & 0.318 & 0 & 0 & 0 & 0 & 0 & 0 & 0 & 0 & 0 & 0 \\
\hline 1973 & 0.099 & 0.886 & 0.759 & 0.318 & 0 & 0 & 0 & 0 & 0 & 0 & 0 & 0 & 0 & 0 \\
\hline 1974 & 0.099 & 0.759 & 0.759 & 0.318 & 0.276 & 0.276 & 0 & 0 & 0 & 0 & 0 & 0 & 0 & 0 \\
\hline 1975 & 0 & 0 & 0.276 & 0.318 & 0.77 & 0.687 & 0.318 & 0 & 0 & 0 & 0 & 0 & 0 & 0 \\
\hline 1976 & 0 & 0 & 0 & 0 & 0.655 & 0.687 & 0.758 & 0 & 0.149 & 0.397 & 0 & 0 & 0 & 0 \\
\hline 1977 & 0 & 0 & 0 & 0.655 & 0.758 & 0.687 & 0.758 & 0 & 0 & 0 & 0 & 0 & 0 & 0 \\
\hline 1978 & 0 & 0 & 0 & 0 & 0.655 & 0.687 & 0.758 & 0 & 0.149 & 0.397 & 0 & 0 & 0 & 0 \\
\hline 1979 & 0 & 0 & 0 & 0.397 & 0.397 & 0.397 & 0.397 & 0 & 0.149 & 0.572 & 0 & 0 & 0 & 0 \\
\hline 1980 & 0 & 0 & 0 & 0 & 0.149 & 0.149 & 0 & 0 & 0.62 & 0.62 & 0 & 0.62 & 0 & 0 \\
\hline 1981 & 0 & 0 & 0 & 0 & 0 & 0 & 0 & 0 & 0.834 & 0.62 & 0 & 0.834 & 0 & 0 \\
\hline 1982 & 0 & 0 & 0 & 0 & 0.95 & 0.687 & 0 & 0 & 0 & 0.149 & 0 & 0 & 0 & 0 \\
\hline 1983 & 0 & 0 & 0 & 0 & 0.655 & 0.687 & 0.758 & 0 & 0.149 & 0.397 & 0 & 0 & 0 & 0 \\
\hline 1984 & 0 & 0 & 0 & 0 & 0.655 & 0.687 & 0.758 & 0 & 0.149 & 0.397 & 0 & 0 & 0 & 0 \\
\hline 1985 & 0 & 0 & 0 & 0.655 & 0.758 & 0.687 & 0.758 & 0 & 0 & 0 & 0 & 0 & 0 & 0 \\
\hline 1986 & 0 & 0 & 0 & 0.655 & 0.758 & 0.687 & 0.758 & 0 & 0 & 0 & 0 & 0 & 0 & 0 \\
\hline 1987 & 0 & 0 & 0 & 0 & 0 & 0 & 0 & 0 & 0.149 & 0.834 & 0 & 0 & 0 & 0 \\
\hline 1988 & 0 & 0 & 0 & 0 & 0 & 0 & 0 & 0 & 0.834 & 0.62 & 0 & 0.834 & 0 & 0 \\
\hline 1989 & 0 & 0 & 0 & 0 & 0 & 0 & 0 & 0 & 0 & 0 & 0 & 0 & 0.847 & 0.847 \\
\hline 1990 & 0 & 0 & 0 & 0 & 0 & 0 & 0 & 0 & 0 & 0 & 0 & 0 & 0.977 & 0.876 \\
\hline 1991 & 0 & 0 & 0 & 0 & 0 & 0 & 0 & 0 & 0 & 0 & 0 & 0 & 0.977 & 0.876 \\
\hline 1992 & 0 & 0 & 0 & 0 & 0 & 0 & 0 & 0 & 0 & 0 & 0 & 0 & 0.977 & 0.876 \\
\hline 1993 & 0 & 0 & 0 & 0 & 0 & 0 & 0 & 0 & 0 & 0 & 0 & 0 & 0.977 & 0.876 \\
\hline
\end{tabular}

By choosing the fuzzified enrollments with the highest membership for each year from Table 2, the results as shown in Table 3 were produced.

Table 3. The Fuzzified Enrollment Output as in Table 2

\begin{tabular}{|c|c|c|c|}
\hline Year & Fuzzified Output & Year & Fuzzified Output \\
\hline 1972 & $\tilde{F}_{2}$ & 1983 & $\tilde{F}_{7}$ \\
\hline 1973 & $\tilde{F}_{2}$ & 1984 & $\tilde{F}_{7}$ \\
\hline 1974 & $\tilde{F}_{2}$ & 1985 & $\tilde{F}_{5}$ \\
\hline 1975 & $\tilde{F}_{5}$ & 1986 & $\tilde{F}_{5}$ \\
\hline 1976 & $\tilde{F}_{7}$ & 1987 & $\tilde{F}_{10}$ \\
\hline 1977 & $\tilde{F}_{5}$ & 1988 & $\tilde{F}_{9}$ \\
\hline 1978 & $\tilde{F}_{7}$ & 1989 & $\tilde{F}_{13}$ \\
\hline 1979 & $\tilde{F}_{10}$ & 1990 & $\tilde{F}_{13}$ \\
\hline 1980 & $\tilde{F}_{10}$ & 1991 & $\tilde{F}_{13}$ \\
\hline 1981 & $\tilde{F}_{9}$ & 1992 & $\tilde{F}_{13}$ \\
\hline 1982 & $\tilde{F}_{5}$ & 1993 & $\tilde{F}_{13}$ \\
\hline
\end{tabular}

After obtaining the fuzzified enrollment output, we formed another set of fuzzy logical relationships and grouped the FLRs accordingly (see Table 4).
Table 4. The FLRs obtained from fuzzy sets and induced fuzzy sets

\begin{tabular}{|c|}
\hline FLRs from induced fuzzy sets \\
\hline$\tilde{F}_{2} \rightarrow \tilde{F}_{2}, \tilde{F}_{2} \rightarrow \tilde{F}_{5}$ \\
$\tilde{F}_{5} \rightarrow \tilde{F}_{5}, \tilde{F}_{5} \rightarrow \tilde{F}_{7}, \tilde{F}_{5} \rightarrow \tilde{F}_{10}$ \\
$\tilde{F}_{7} \rightarrow \tilde{F}_{5}, \tilde{F}_{7} \rightarrow \tilde{F}_{7}, \tilde{F}_{7} \rightarrow \tilde{F}_{10}$ \\
$\tilde{F}_{9} \rightarrow \tilde{F}_{5}, \tilde{F}_{9} \rightarrow \tilde{F}_{13}$ \\
$\tilde{F}_{10} \rightarrow \tilde{F}_{9}, \tilde{F}_{10} \rightarrow \tilde{F}_{10}$ \\
$\tilde{F}_{13} \rightarrow \tilde{F}_{13}$
\end{tabular}

By using the arithmetic rules, the forecasted enrollments could be obtained.

\section{Results and Discussion}

In this section, we present the forecasted enrollments using the proposed model and compare them with the fuzzy time series forecasting model based on fuzzy sets. The forecasted enrollments are given in Table 5. 
Table 5. Forecasted enrollments at the University of Alabama

\begin{tabular}{|c|c|c|c|}
\hline Year & $\begin{array}{c}\text { Actual } \\
\text { Enrollment }\end{array}$ & $\begin{array}{c}\text { Forecasted } \\
\text { Enrollments } \\
\text { Based on } \\
\text { Fuzzy Sets } \\
\end{array}$ & \begin{tabular}{|c|} 
Forecasted \\
Enrollments \\
Based on Induced \\
Fuzzy Sets \\
\end{tabular} \\
\hline 1972 & 13563 & - & - \\
\hline 1973 & 13867 & 14562.5 & 14562.5 \\
\hline 1974 & 14696 & 14562.5 & 14562.5 \\
\hline 1975 & 15460 & 14562.5 & 14562.5 \\
\hline 1976 & 15311 & 16104.25 & 16027.83 \\
\hline 1977 & 15603 & 16104.25 & 16027.83 \\
\hline 1978 & 15861 & 16104.25 & 16027.83 \\
\hline 1979 & 16807 & 16104.25 & 16027.83 \\
\hline 1980 & 16919 & 16500 & 16666.75 \\
\hline 1981 & 16388 & 16500 & 16666.75 \\
\hline 1982 & 15422 & 17062.5 & 17062.5 \\
\hline 1983 & 15497 & 16104.25 & 16027.83 \\
\hline 1984 & 15145 & 16104.25 & 16027.83 \\
\hline 1985 & 15163 & 16104.25 & 16027.83 \\
\hline 1986 & 15984 & 16104.25 & 16027.83 \\
\hline 1987 & 16859 & 16104.25 & 16027.83 \\
\hline 1988 & 18150 & 16500 & 16666.75 \\
\hline 1989 & 18970 & 17062.5 & 17062.5 \\
\hline 1990 & 19328 & 18750 & 18750 \\
\hline 1991 & 19337 & 18750 & 18750 \\
\hline 1992 & 18876 & 18750 & 18750 \\
\hline 1993 & & 18750 & 18750 \\
\hline
\end{tabular}

To evaluate the performance of the forecasting model, we calculated the mean square error (MSE), root mean square error (RMSE), mean absolute error (MAE), and mean absolute percentage error (MAPE).

Table 6. The MSE, RMSE, MAE, and MAPE for the forecasted enrollments

\begin{tabular}{|c|c|c|}
\hline $\begin{array}{c}\text { Types of } \\
\text { Error }\end{array}$ & $\begin{array}{c}\text { Forecasted Enrollments } \\
\text { Based on Fuzzy Sets }\end{array}$ & $\begin{array}{c}\text { Forecasted } \\
\text { Enrollment Based on } \\
\text { Induced Fuzzy Sets }\end{array}$ \\
\hline MSE & 769925.3406 & 723395.4344 \\
\hline RMSE & 877.4538966 & 850.526563 \\
\hline MAE & 717.9375 & 690.4958333 \\
\hline MAPE & 0.04354246 & 0.041827848 \\
\hline
\end{tabular}

In reference to all error values calculated in Table 6 , the forecasted enrollments based on fuzzy sets induced by intuitionistic fuzzy sets showed better performance compared to the forecasted enrollments based on fuzzy sets alone. This indicates that the intuitionistic fuzzy sets play a better role in forecasting time series data rather than the classical fuzzy sets.

\section{Conclusion}

Overall, by comparing the MSE, RMSE, MAE, and MAPE for each model, it can be deduced that the forecasting model based on the induced fuzzy sets obtained from intuitionistic fuzzy sets performs better than the model that is solely based on fuzzy sets.

Since the intuitionistic fuzzy sets consider both the degree of membership and non-membership, the model that uses intuitionistic fuzzy sets as a basis could manage to consider the degree of hesitation in forecasting. This is consistent with the result obtained in [26], which states that the utilization of intuitionistic fuzzy sets is more useful and able to give a good decision. This supports the fact that an intuitionistic fuzzy set is indeed a generalization of a fuzzy set.

\section{Acknowledgement}

The authors would like to thank Universiti Teknologi MARA for financially supporting the publication of this article under Lestari Covid-19 Research Grant UiTM 600-RMC/LESTARI COVID/5/3(038/2020).

\section{REFERENCES}

[1] Q. Song, B. S. Chissom. Fuzzy Time Series and its Model, Fuzzy Sets and Systems, 54, 269-277, 1993.

[2] Q. Song, B. S. Chissom. Forecasting Enrollments with Fuzzy Time Series - Part I, Fuzzy Sets and Systems, 54, 1-9, 1993.

[3] Q. Song, B. S Chissom. Forecasting Enrollments with Fuzzy Time Series - Part II, Fuzzy Sets and Systems, Vol.62, 1-8, 1994.

[4] S. M. Chen. Forecasting Enrollments Based on Fuzzy Time Series, Fuzzy Sets and Systems, Vol.81, 311-319, 1996.

[5] K. Huarng. Heuristic Models of Fuzzy Time Series for Forecasting, Fuzzy Sets and Systems, Vol.123, 369-386, 2001.

[6] K. Huarng. Effective Lengths of Intervals to Improve Forecasting in Fuzzy Time Series, Fuzzy Sets and Systems, 123, 387-394, 2001.

[7] S. -M. Chen. Forecasting Enrollments Based on High-Order Fuzzy Time Series, Cybernetics and Systems, Vol.33, 1-16, 2002.

[8] H. -T. Liu. An Improved Fuzzy Time Series Forecasting Method using Trapezoidal Fuzzy Numbers, Fuzzy Optimisation and Decision Making, Vol.6, 63-80, 2007.

[9] S. M. Chen, X. Y. Zou, G. C. Gunawan. Fuzzy time series forecasting based on proportions of intervals and particle swarm optimization techniques, Information Sciences, 500, 127-139, 2019. 
[10] P. C. De Lima e Silva, C. A. Severiano, M. A. Alves, R. Silva, M. W. Cohen, F. G. Guimarães. Forecasting in non-stationary environments with fuzzy time series, Applied Soft Computing, Vol. 97, 106825, 2020.

[11] A. K. Rana. Comparative Study on Fuzzy Models for Crop Production Forecasting, Mathematics and Statistics, Vol.8, No.4, 451-457, 2020.

[12] K. Atanassov. Intuitionistic Fuzzy Sets, Fuzzy Sets and Systems, Vol.20, 87-96, 1986.

[13] O. Castillo, A. Alanis, M. Garcia, H. Arias. An Intuitionistic Fuzzy System for Time Series Analysis in Plant Monitoring and Diagnosis, Applied Soft Computing, Vol.7, 1227-1233, 2007.

[14] B. P. Joshi, S. Kumar. Intuitionistic Fuzzy Sets Based Method for Fuzzy Time Series Forecasting, Cybernetics and Systems, Vol.43, 34-47, 2012.

[15] S. S. Gangwar, S. Kumar. Probabilistic and Intuitionistic Fuzzy Sets-based Method for Fuzzy Time Series Forecasting, Cybernetics and Systems, Vol.45, 349-361, 2014.

[16] S. Kumar, S. S. Gangwar. A Fuzzy Time Series Forecasting Method Induced by Intuitionistic Fuzzy Sets, International Journal of Modelling, Simulation and Scientific Computing, Vol.6, No.4, 1-23, 2015.

[17] X. Fan, Y. Lei, Y. Wang. Adaptive Partition Intuitionistic Fuzzy Time Series Forecasting Model, Journal of Systems Engineering and Electronics, Vol.28, No.3, 585-596, 2017.

[18] K. Bisht, D. K. Joshi, S. Kumar. Dual Hesitant Fuzzy Set-based Intuitionistic Fuzzy Time Series Forecasting, Ambient Communications and Computer Systems, Vol.696, 317-329, 2018.
[19] K. K. Gupta, S. Kumar. Fuzzy Time Series Forecasting Method using Probabilistic Fuzzy Sets, Advanced Computing and Communication Technologies, In Mandal, J. K., Bhattacharyya, D. \& Auluck, N. (Eds.), (pp. 35-43). Singapore: Springer Singapore, 2019.

[20] S. S. G. Abhishekh, S. R. Singh. A score function-based method of forecasting using intuitionistic fuzzy time series, New Mathematics and Natural Computation, Vol.14, No.1, 91-111, 2018

[21] K. Bisht, S. Kumar. Intuitionistic fuzzy set-based computational method for financial time series forecasting, Fuzzy Information and Engineering, Vol.10, No.3, 307-323, 2019.

[22] V. Atanassova, S. Sotirov. A new formula for de-i-fuzzification of intuitionistic fuzzy sets, Notes on Intuitionistic Fuzzy Sets, Vol.18, No.3, 49-51, 2012.

[23] A. Ban, J. Kacprzyk, K. Atanassov. On de-I-fuzzification of intuitionistic fuzzy sets, Comptes Rendus de l'Academie bulgare des Sciences, Vol.61, No.12, 1535-1540, 2008.

[24] A. Q. Ansari, J. Philip, S. A. Siddiqui, J. A. Alvi, Fuzzification of Intuitionistic Fuzzy Sets, International Journal of Computational Coginition, Vol.8, No.3, 90-91, 2010.

[25] S. M. Chen, C. C. Hsu, A New Method to Forecast Enrollments using Fuzzy Time Series, International Journal of Applied Science and Engineering, Vol.2, No.3, 234-244, 2004.

[26] P. Arora, V. P. Tomar. Measuring Given Partial Information about Intuitionistic Fuzzy Sets, Mathematics and Statistics, Vol.8, No.6, 665-670, 2020. 